\title{
Article
}

\section{Resource Allocation Using Reconfigurable Intelligent Surface (RIS)-Assisted Wireless Networks in Industry 5.0 Scenario}

\author{
Sonain Jamil ${ }^{1, *(\mathbb{D}, \text { MuhibUr Rahman }}{ }^{2, *(\mathbb{D})}$, Muhammad Sohail Abbas ${ }^{3}$ (D) and Fawad $^{4}$ (D) \\ 1 Department of Electronics Engineering, Sejong University, Seoul 05006, Korea \\ 2 Department of Electrical Engineering, Polytechnique Montreal, Montreal, QC H3T 1J4, Canada \\ 3 School of Electrical Engineering and Computer Science (SEECS), National University of Sciences and \\ Technology (NUST), Islamabad 44000, Pakistan; mabbas.mscs20seecs@seecs.edu.pk \\ 4 Department of Telecommunication Engineering, University of Engineering and Technology (UET), \\ Taxila 47080, Pakistan; engr.fawad@students.uettaxila.edu.pk \\ * Correspondence: sonainjamil@sju.ac.kr (S.J.); muhibur.rahman@polymtl.ca (M.R.)
}

Citation: Jamil, S.; Rahman, M.R.; Abbas, M.S.; Fawad. Resource Allocation Using Reconfigurable Intelligent Surface (RIS)-Assisted Wireless Networks in Industry 5.0 Scenario. Telecom 2022, 3, 163-173. https://doi.org/10.3390/ telecom3010011

Academic Editor: Sotirios K. Goudos

Received: 17 January 2022

Accepted: 17 February 2022

Published: 1 March 2022

Publisher's Note: MDPI stays neutral with regard to jurisdictional claims in published maps and institutional affiliations.

Copyright: (C) 2022 by the authors. Licensee MDPI, Basel, Switzerland. This article is an open access article distributed under the terms and conditions of the Creative Commons Attribution (CC BY) license (https:// creativecommons.org/licenses/by/ $4.0 /)$.

\begin{abstract}
Mobile communication networks evolved from first-generation (1G) to sixth-generation (6G) and the requirement for quality of services (QoS) and higher bandwidth increased. The evolvement of $6 \mathrm{G}$ can be deployed in industry 5.0 to fulfill the future industry requirement. However, deploying 6G in industry 6.0 is very challenging, and installing a reconfigurable intelligent surface (RIS) is an efficient solution. RIS contains the passive elements which are programmed for the tuning of a wireless channel. We formulate an optimization problem to allocate resources in the RIS-supported network. This article presents a mixed-integer non-linear programable problem (MINLP) considering the industry 5.0 scenario and proposes a novel algorithm to solve the optimization problem. We obtain the $\epsilon$ optimal solution using the proposed algorithm. The proposed algorithm is evaluated in energy efficiency (EE), throughput, latency, and channel allocation. We compare the performance of several algorithms, and the proposed algorithm outperforms all the algorithms.
\end{abstract}

Keywords: reconfigurable intelligent surface; optimization; 6G; industry 5.0; resource allocation

\section{Introduction}

In the modern era of big data, the demand for higher data rates, low latency, and reliable communication is increasing daily [1]. In the past few years, the number of users is increasing drastically as mobile network communication evolved from first-generation (1G) to fifth-generation (5G) [2]. 1G supported voice calls only, 2G kept message services while 3G had internet support. 4G grew in the 2010s and supported mobile broadband (MBB). In the 2020s, 5G evolved and offered mobile broadband (eMBB), ultra-reliable low latency (URLLC), and massive machine-type communication (mMTC) services. Nowadays, the sixth generation $(6 \mathrm{G})$ is developing and offers intelligence and sensing technologies. These technologies require more bandwidth and data rates. Figure 1, shows the evolution of mobile communication from $2 \mathrm{G}$ to $6 \mathrm{G}$.

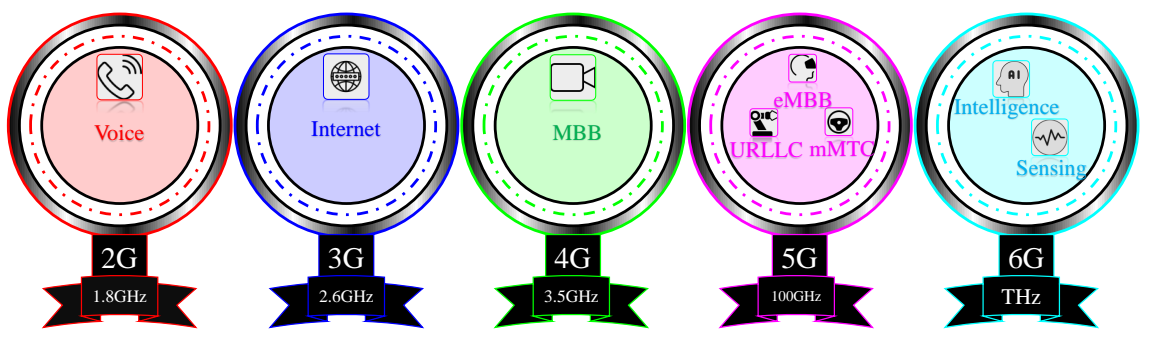

Figure 1. Evolution of mobile network from $2 \mathrm{G}$ to $6 \mathrm{G}$. 
Moreover, the use of mobile networks in the industry has also increased, and to fulfill the stringent requirements of industry 5.0 and beyond, the research to deploy nextgeneration (6G) networks has started. Industry 5.0 is based on human-centric solutions and supports mass customization. Figure 2 shows the industrial evolution. The primary requirements for industry 5.0 are high energy efficiency (EE), low latency, high spectral efficiency (SE), and high throughput [3]. The solution to fulfill all these requirements is deploying 6G in industry 5.0. However, the deployment of 6G in the industry is quite challenging. Due to non-line of sight (NLOS), the EE and throughput of the network decrease. NLOS also gives rise to multi-path fading, attenuation, and interference due to reflection and refraction. To overcome this problem, reconfigurable intelligent surface (RIS) is the primary solution [4]. RIS reduces attenuation and improves network performance [5].
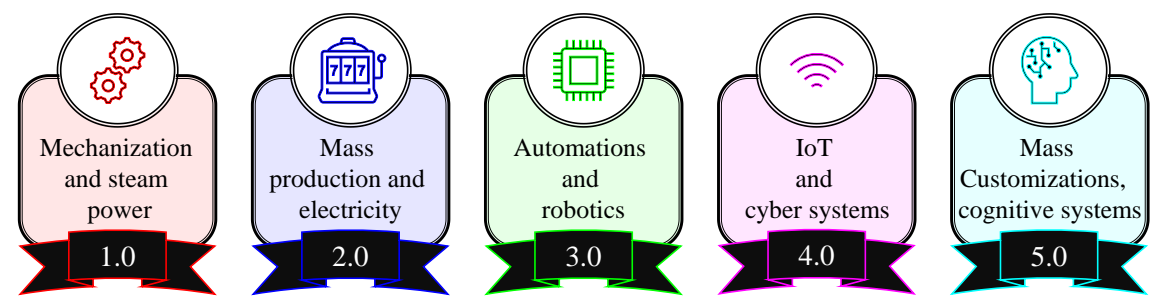

Figure 2. Evolution of industry from 1.0 to 5.0.

RIS consists of electromagnetic (EM) materials and possesses EM properties. These are thin sheets deployed on the buildings, walls, and machines. The EM nature of the RIS helps to monitor the medium of the whole network and the mode of operation of RIS, which is full-duplex [6]. The deployment of RIS is also cost-effective because it is a passive device. PIN diode controls the phase shift $(\Theta)$ of RIS [7] and helps to achieve a particular phase by switching function [8].

When the state of the PIN diode is low the incoming energy $(h v)$ passes through it due to its installation on the frequency selective surface (FSS). Whereas, when the state of the diode is high most of $h v$ is reflected. A RIS-assisted network base station (BS) transmits a signal to RIS and RIS improves the characteristics of the incoming signal which helps in improving the overall performance of the network [9-13].

Inspired by the numerous benefits of RIS-assisted networks, we formulate an optimization problem to enhance EE, SE, throughput and minimize the latency of the network in an industry 5.0 scenario. We propose a novel algorithm to solve the optimization problem. Following are the primary contributions of this article.

1. We formulate an optimization problem in the RIS-assisted wireless network in the industry 5.0 scenario.

2. We propose a novel algorithm to solve the optimization problem and evaluate EE, SE, throughput, and latency.

3. We allocate resources and evaluate the channel mode.

4. We compare the performance of the proposed algorithm with others.

\section{Related Work}

RIS-assisted networks are widely deployed in the 6G. In [6], the authors studied the use of RIS and multiple-input single-output (MISO) technologies. They proposed an alternating minimization approach to increase EE of the system under constraints of no beamforming and NLOS between transmitter $\left(T_{x}\right)$ and receiver $\left(R_{x}\right)$. Similarly, in [14], the authors demonstrated the use of a RIS-assisted MISO network to increase the sum rate. They optimized the continuous $\Theta$ of RIS and beamformer of $T_{x}$.

The authors of [15] explored the use of RIS with single input single output (SISO) network assuming channel state information (CSI) and network achieved a higher signalto-noise ratio (SNR). In [16], the authors also used RIS with MISO and maximized the signal-to-interference and noise ratio (SINR) of the network. In [17], the SNR of the network 
was improved using RIS in the single cell. Furthermore, Le et al. proposed an alternating descent gradient algorithm to maximize the EE of the network. The algorithm showed a significant gain in the EE of the cell-free network.

In [18], Lee et al. applied deep reinforcement learning (DRL) to improve the EE. They showed that the EE improved up to $77 \%$ by increasing the number of RIS elements. Similarly, in [19], the authors proposed the Dinkelbach method to enhance the EE of the device-to-device (D2D) network. The algorithm showed significant improvements in EE of the D2D network. In [20], the authors used RIS surface to enhance the security of the physical layer in the $6 \mathrm{G}$ wireless network. The performance showed a significant improvement in security using RIS. Similarly, Odeyemi et al. in [21] optimized the power of the wireless network using RIS surface. The use of RIS in next-generation wireless networks is surveyed in [22].

Moreover, You et al. in [23] used an accelerated projected gradient algorithm to achieve higher EE and SE in a RIS-assisted multiple-input multiple-output (MIMO) network. Similarly, in [24], the authors used the power consumption method to enhance the data rate and EE of the network. In [25], the authors used an optimization algorithm to enhance the throughput and EE of the RIS-assisted network. Similarly, in [26], a Q-learning based approach is adopted to manage interference in the heterogeneous networks (HetNets). In [27], the authors surveyed the game-theoretical approaches for the virtualization of the cellular network.

Table 1 summarizes the existing RIS-assisted networks. In this article, we propose an outer approximation algorithm (OAA) to maximize EE, SE, throughput and minimize the latency of the RIS-assisted network.

Table 1. Summary of Existing RIS-assisted Wireless Networks.

\begin{tabular}{|c|c|c|c|c|c|c|c|}
\hline \multirow{2}{*}{\multicolumn{2}{|c|}{ Research Approach }} & \multicolumn{5}{|c|}{ Objective } & \multirow{2}{*}{ Contributions } \\
\hline & & EE & Throughput & SE & Latency & Channel Allocation & \\
\hline$[6]$ & $\begin{array}{l}\text { Gradient descent } \\
\text { search }\end{array}$ & $\checkmark$ & $\times$ & $\times$ & $\times$ & $\times$ & $300 \%$ higher $\mathrm{EE}$ \\
\hline [28] & $\begin{array}{l}\text { Alternating } \\
\text { descent algorithm }\end{array}$ & $\checkmark$ & $\times$ & $x$ & $\times$ & $\times$ & $\begin{array}{l}\text { Signification gain in EE of cell- } \\
\text { free network }\end{array}$ \\
\hline [18] & DRL & $\checkmark$ & $\times$ & $x$ & $\times$ & $x$ & $\begin{array}{l}\text { EE improved up to } 77 \% \text { by in- } \\
\text { creasing the number of RIS ele- } \\
\text { ment }\end{array}$ \\
\hline [19] & $\begin{array}{l}\text { Dinkelbach } \\
\text { method }\end{array}$ & $\checkmark$ & $x$ & $\times$ & $\times$ & $\times$ & $\begin{array}{l}\text { The algorithm provided a signif- } \\
\text { icant improvement in the EE of } \\
\text { D2D network }\end{array}$ \\
\hline [23] & $\begin{array}{l}\text { Accelerated pro- } \\
\text { jected gradient }\end{array}$ & $\checkmark$ & $x$ & $\checkmark$ & $\times$ & $\times$ & $\begin{array}{l}\text { Achieved higher EE in MIMO } \\
\text { network }\end{array}$ \\
\hline [24] & $\begin{array}{l}\text { Power consump- } \\
\text { tion method }\end{array}$ & $\checkmark$ & $\times$ & $\times$ & $\times$ & $\times$ & $\begin{array}{l}\text { RIS network surpasses relay one } \\
\text { in terms of both rate and EE }\end{array}$ \\
\hline [25] & $\begin{array}{l}\text { Approximation } \\
\text { Algorithm }\end{array}$ & $\checkmark$ & $\checkmark$ & $\times$ & $\times$ & $\times$ & $\begin{array}{l}\text { Maximization of throughput and } \\
\text { EE in the RIS-assisted wireless } \\
\text { network }\end{array}$ \\
\hline $\begin{array}{l}\text { This } \\
\text { paper }\end{array}$ & OAA & $\checkmark$ & $\checkmark$ & $\checkmark$ & $\checkmark$ & $\checkmark$ & $\begin{array}{l}\text { Improved throughput, EE, SE of } \\
\text { the network; minimized latency }\end{array}$ \\
\hline
\end{tabular}

\section{Scenario and Problem Formulation}

In this article, an industry 5.0 scenario in a company ABC Limited (Ltd) is supposed. In this scenario, a RIS-based wireless network is deployed. ABC Ltd has several departments and has more than 300 employees. The company also has automation and robotics support. 
The company requires high throughput, low latency, and high EE. The complete network of ABC Ltd. is shown in Figure 3.

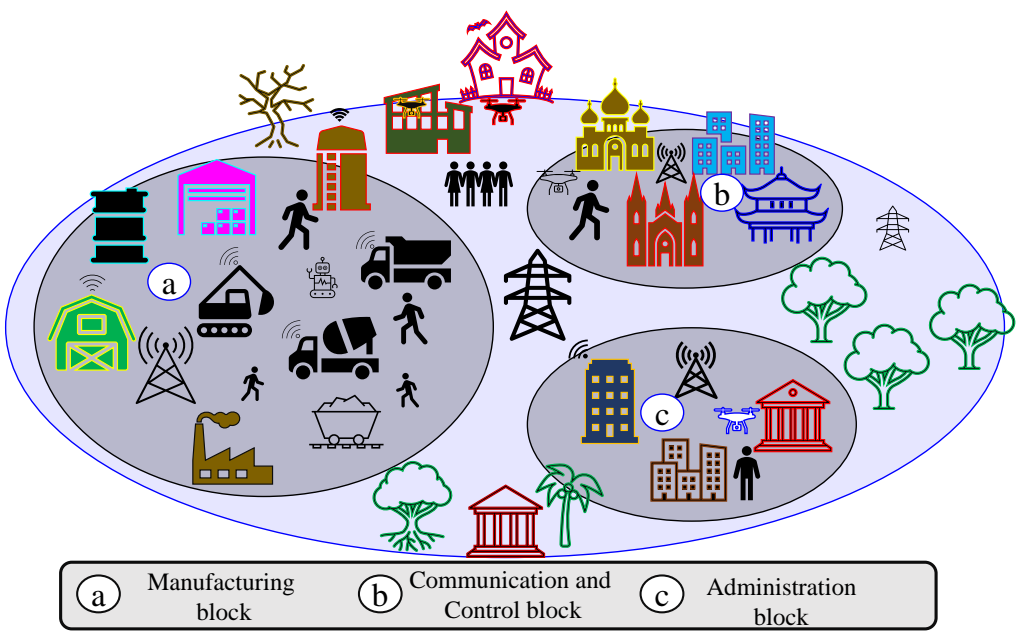

Figure 3. ABC Ltd. Scenario.

\subsection{RIS-Assisted Scenario}

Consider a manufacturing block of ABC Ltd. as shown in Figure 4, one BS has $\mathbb{N}$ antennas. RIS connects BS, and the plane of BS is $\mathrm{YZ}$, whereas the plane of RIS is XZ. There are $\mathbb{K}$ reflecting elements of RIS. This surface serves $\mathbb{X}$ users. There is no line of sight (LOS) between the user $\left(U_{E}\right)$ and the BS due to hurdles. $\mathbb{C}_{1}$ is the channel between BS and RIS, and $\mathbb{C}_{2}$ is the channel between the RIS and the $i$ th $U_{E} . \Theta$ is the phase shift matrix of the RIS.

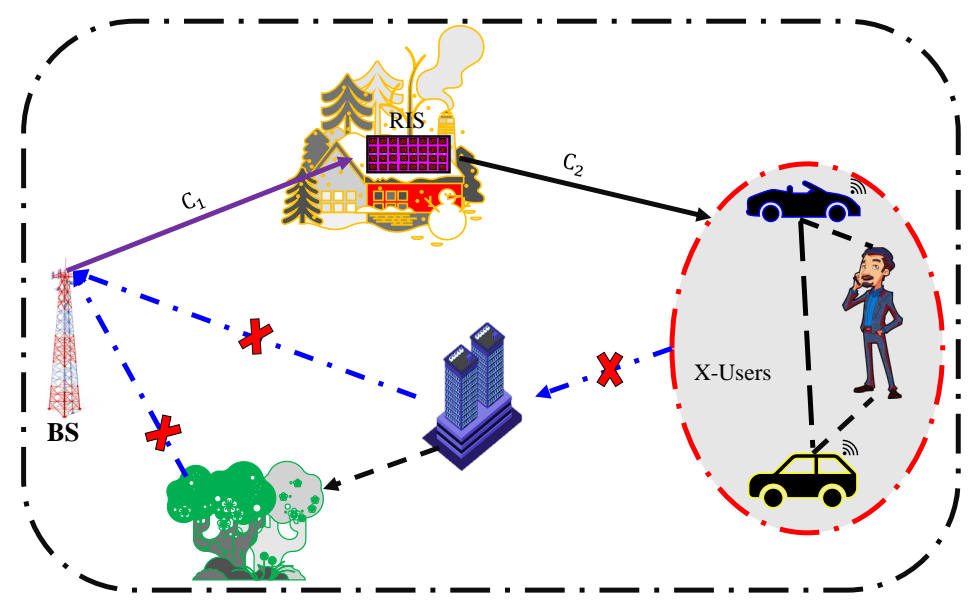

Figure 4. RIS-aided network scenario.

\subsection{Problem Formulation}

In the scenario shown in Figure 4 , we have $i$ users with $i=\{1,2,3, \ldots, \mathbb{X}\}$ and we can find the received signal with Equation (1) [6].

$$
\mathbb{R}_{u}=\mathbb{H}_{2} \times \Theta \times \mathbb{H}_{1 \chi}+\eta_{u}
$$

where $\mathbb{H}_{1}$ denotes the channel matrix between BS and the RIS and $\mathbb{H}_{1} \in \mathbb{C}^{\mathbb{K} * \mathbb{N}}, \mathbb{H}_{2} \in \mathbb{C}^{1 * \mathbb{K}}$ denotes channel matrix between RIS and the user $u$. $\Theta$ represents the actual phase shift, 
and $\eta_{u}$ denotes the noise. $\eta_{u}$ is generated by transmission. $\chi$ represents the transmitted signal from the BS and can be evaluated by Equation (2) [6].

$$
\chi=\sum_{u=1}^{\mathbb{X}} \sqrt{p_{u} \times g_{u} \times s_{u}}
$$

where $g_{u} \in \mathbb{C}^{* 1}$ denotes precoding vector. $s_{u}$ denotes the unit-power from the discrete constellation set and $p_{u}$ denotes the unit-power from total power $\left(P_{T x}\right)$. The SINR $(\gamma)$ can be calculated by Equation (3) [6].

$$
\gamma_{i} \triangleq \frac{p_{i} \times\left|h_{2, i} \times \Theta \times \mathbb{H}_{1} \times g_{i}\right|^{2}}{\sum_{j=1, j \neq i}^{K} p_{i} \times\left|h_{2, i} \times \Theta \times \mathbb{H}_{1} g_{j}\right|^{2}+\sigma^{2}}
$$

Using Equation (3), we can calculate the throughput of the network with help of Shannon capacity formula as in Equation (4).

$$
\text { Throughput }(\Omega)=\sum_{i=1}^{\mathbb{X}} \log _{2}\left(1+\gamma_{i}\right)
$$

where $\gamma_{i}$ is the SINR of $i$ th users. The instantaneous power $P_{i}$ can be calculated by (5).

$$
\mathbb{P}_{i}=\mathbb{P}_{U E}+\mathbb{P}_{B S}+\mathbb{P}_{R I S}
$$

Here $\mathbb{P}_{U E}$ denotes static power of $U_{E}, \mathbb{P}_{B S}$ represents the static power of BS and $\mathbb{P}_{R I S}$ shows total hardware static power absorbed by RIS. Equation (6) gives the power of RIS with $\mathbb{K}$ reflecting element.

$$
P_{R I S}=\mathbb{P}(\theta) * \mathbb{K}
$$

where $\mathbb{P}(\theta)$ is the dissipated power of phase-shifter. The total power $\left(P_{T}\right)$ of the network can be calculated by Equation (7) [6].

$$
\mathbb{P}_{T}=\sum_{i=1}^{\mathbb{X}} \mathbb{P}_{U E}+\sum_{i=1}^{\mathbb{N}} \mathbb{P}_{B S}+\sum_{i=1}^{\mathbb{K}} \mathbb{P}_{R I S}
$$

The $\mathbb{E} \mathbb{E}$ of the network can is the ratio of data rate and $P_{T}$. Mathematically it can written by Equation (8a). This objective function is subject to constraints from (8b) to (8g).

$$
\begin{gathered}
\mathbb{E}_{\text {max }}=\frac{\Omega}{P_{T}} \\
\text { subject to: } \quad P_{\text {min }} \leq P_{c} \\
S I N R_{\text {min }} \leq S I N R_{c} \\
0 \leq P_{c} \leq P^{\text {max }} \\
R^{c} \geq R^{\text {min }}=\sum_{c \in C} X_{c}^{u} c p_{c} \geq \sum_{c \in C} X_{c} C p^{u} \\
p_{c}^{u} \leq x_{c}^{u} P_{c} \quad c \in C ; C=\{c 1, c 2\}, u \in U ; U=\{1,2,3, \ldots, u\} \\
p_{c}^{u} \geq 0
\end{gathered}
$$

1. The power of $c$ th connection must be greater than or equal to minimum power $(8 b)$.

2. The SINR of $c$ th connection must be greater than or equal to minimum SINR (8c).

3. The power of $c$ th connection must be greater than or equal to zero and less than or equal to maximum $(8 \mathrm{~d})$.

4. The data rate of $c$ th connection must be greater than or equal to minimum data rate (8e). 
5. No power is allocated to non-connected $U_{E} \cdot P_{c}$ denotes the maximum transmission power of $c$ th link while $p_{c}^{u}$ represents the $c$ th channel power having $u U_{E}(8 \mathrm{f})$.

6. The minimum power of $c$ th $U_{E}$ must be greater than or equal to $0(8 \mathrm{~g})$.

The upcoming section briefly explains the proposed algorithm to optimize the problem in Equation (8a).

\section{Proposed Method}

We propose OAA to solve the problem in (8a). The algorithm starts by relaxing integer variables to obtain a non-linear (NLP) subproblem. Applying linearization to non-linear constraints converts the NLP subproblem into the master mixed-integer problem (MIP). Then the feasibility of the MIP is evaluated. If MIP is infeasible, the algorithm stops, and if it is feasible, then fix integer variables and solve the NLP problem again. Add the integer elimination constraints so that the same solution is not obtained in each iteration. The pseudo code of algorithm is shown in Algorithm 1.

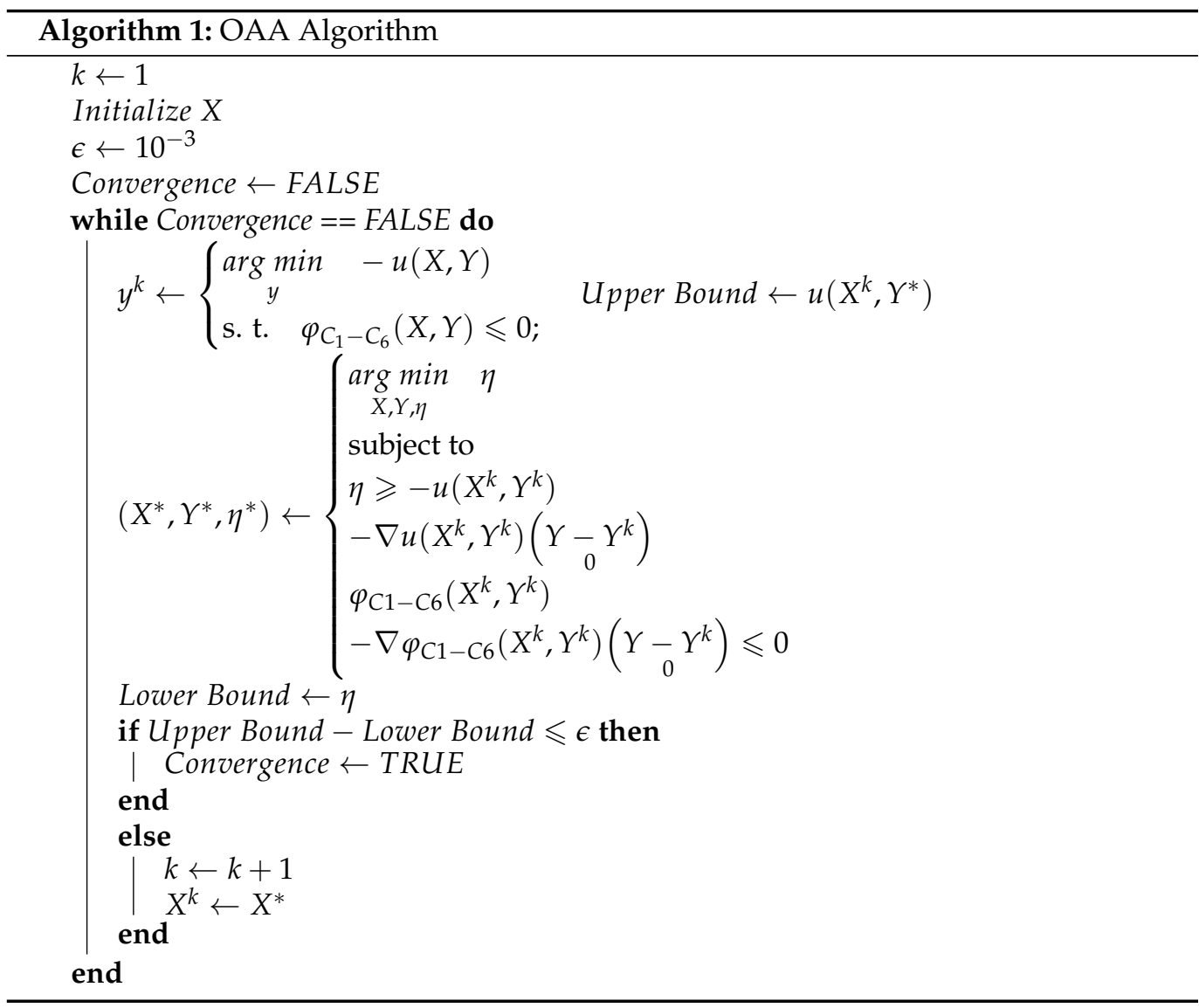

\subsection{Description of OAA Algorithm}

Suppose $U$ be the objective function as in (8a) subject to $\varphi_{C_{1}-C_{6}}(8 \mathrm{~b})$ to $(8 \mathrm{~g})$ and $Y=\left\{\mathbb{P}_{U E}, \mathbb{P}_{B S}, \mathbb{P}_{R I S}\right\}$. Whereas $X=x \cup Y$. The following hypothesis are applied to (8a).

1. $Y$ is the set with objective $U$. It possesses constraints $\varphi_{C_{1}}-C_{6}$.

2. $U$ and $\varphi_{C_{1}-C_{6}}$ are continuous differentiable.

3. Optimal solution obtained by fixing the $X$ satisfies all the constraints.

4. If we have solution of $X$, we can obtain the optimal solution of $U$. 
All these hypotheses convert problem (8a) to NLP complex problem, and OAA gives $\epsilon$-optimal solution at convergence. We can achieve the main problem by adjusting $x$. Integer variables of OAA are iterated $\kappa$ th times. The problem can be written as:

$$
\left\{\begin{array}{l}
\underset{Y}{\arg \min }-U(X, Y) \\
\text { s. t. } \quad \varphi_{C_{1}-C_{6}}(X, Y) \leqslant 0
\end{array}\right.
$$

The solution of (9) is set to $Y^{\mathcal{K}}$ and applying the upper and lower limit. We can get the master MIP problem by first converting (9) to integer space and then applying linearization. The problem in integer space $X$ can be written as:

$$
\left\{\begin{array}{l}
\min _{X} \min _{Y}-U\left(X^{\kappa}, Y\right) \\
\text { s. t. } \quad \varphi_{C_{1}-C_{6}}\left(X^{\kappa}, Y\right) \leqslant 0
\end{array}\right.
$$

The master problem in (10) can be represented as:

$$
\min _{X}-\theta(X)
$$

where

$$
\left\{\begin{array}{l}
\theta(X)=\min _{Y}-U\left(X^{\kappa}, Y\right) \\
\text { s. t. } \quad \varphi_{C_{1}-C_{6}}\left(X^{\kappa}, Y\right) \leqslant 0
\end{array}\right.
$$

The equation in (11) is the projection of the (8a) with distinct variables. Then according to OAA linearization is applied to get master MIP problem. The master MIP problem can be written as:

$$
\left\{\begin{array}{l}
\min _{X} \min _{Y}-U\left(X^{\kappa}, Y^{\kappa}\right)-\nabla U\left(X^{\kappa}, Y^{\kappa}\right)\left(\begin{array}{c}
Y-Y^{\kappa} \\
X-X^{\kappa}
\end{array}\right) \\
\text { s. t.: }-\varphi_{C_{1}-C_{6}}\left(X^{\kappa}, Y^{\kappa}\right)-\nabla \varphi_{C_{1}-C_{6}}\left(X^{\kappa}, Y^{\kappa}\right)\left(\begin{array}{c}
Y-Y^{\kappa} \\
X-X^{\kappa}
\end{array}\right)
\end{array}\right.
$$

If the algorithm does not converge then a new variables are introduced and can be written as:

$$
\min _{X, Y, \eta} \eta \text { s.t.: }\left\{\begin{array}{l}
\eta \geqslant-U\left(X^{\kappa}, Y^{\kappa}\right)-\nabla U\left(X^{\kappa}, Y^{\kappa}\right)\left(\begin{array}{l}
Y-Y^{\kappa} \\
X-X^{\kappa}
\end{array}\right) \\
-\varphi_{C_{1}-C_{6}}\left(X^{\kappa}, Y^{\kappa}\right)-\nabla \varphi_{C_{1}-C_{6}}\left(X^{\kappa}, Y^{\kappa}\right)\left(\begin{array}{c}
Y-Y^{\kappa} \\
X-X^{\kappa}
\end{array}\right) \leqslant 0
\end{array}\right.
$$

OAA solves the MINLP problem (8a). For the resources allocation we use Algorithm 2.

\subsection{Resource Allocation}

For successful and reliable communication, each $U_{E}$ needs better resources. We use the following Algorithm 2 to allocate optimal resources to the $U_{E} s$. Initially, the random base stations (BSs) are assigned to the $U_{E} S$, and $U_{E} S$ discovers the nearest BS proximity. $U_{E} S$ and BSs calculate the utility, and blocking is monitored. If the blockage is found, RIS resources are assigned. Then the $U_{E} S$ sends the proposal to the optimal BS, and the utility is calculated if the utility increases the BSs accept proposals, and stable matching is achieved. 


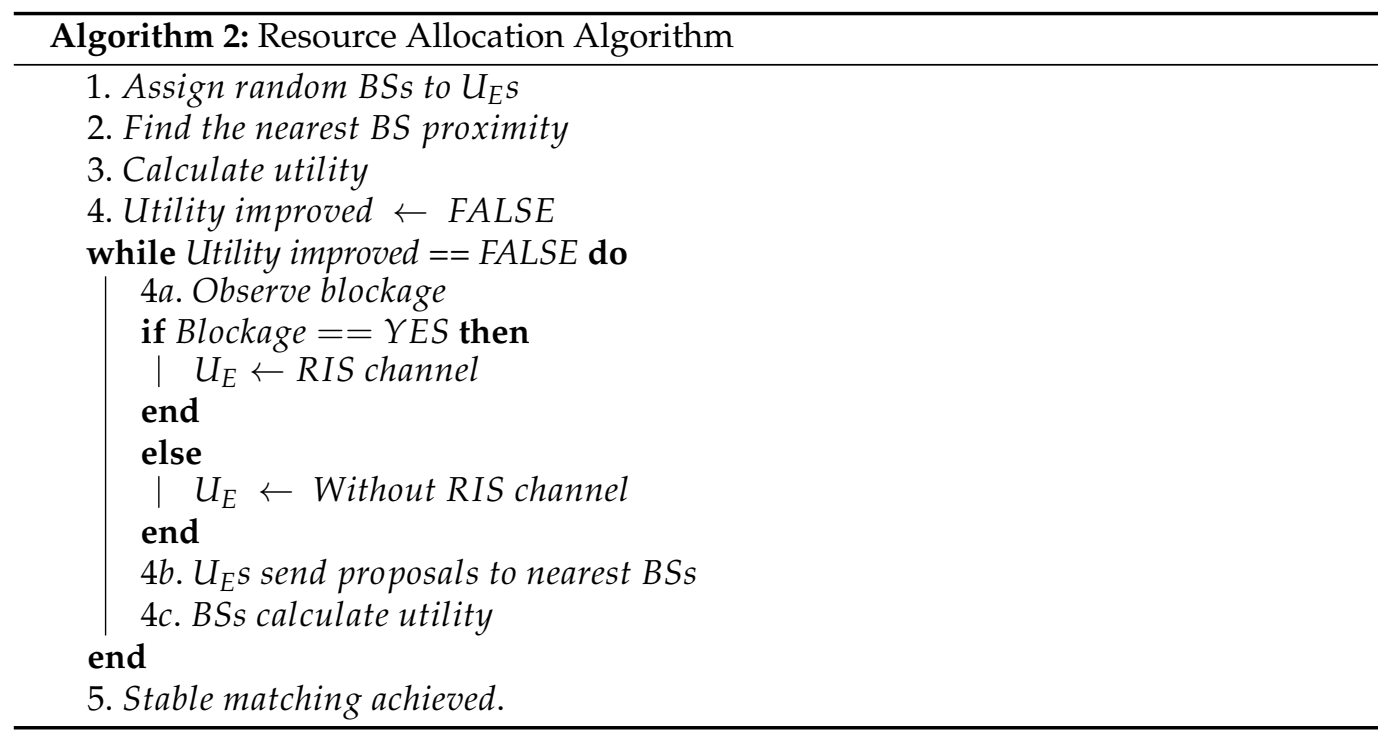

The next section shows the experimental results obtained with OAA.

\section{Experimental Results}

We simulated the network in MATLAB and solved the problem in Equation (8a) using OAA. The value of $P_{B S}$ is $20 \mathrm{dBW}$. $P_{U E}$ and $P_{R I S}$ is $10 \mathrm{dBm}$. The minimum data rate $\left(R_{\min }\right)$ is $0.1 \mathrm{bits} / \mathrm{s} / \mathrm{Hz}$. The maximum number of users $\left(\max _{U E}\right)$ is 300 whereas the minimum number of users $\left(\min _{U E}\right)$ is 2 . The simulation parameters for the network are summarized in Table 2.

Table 2. Simulation Parameters.

\begin{tabular}{lll}
\hline Parameter & Value & Unit \\
\hline$P_{B S}$ & 20 & $\mathrm{dBW}$ \\
\hline$P_{U E}$ & 10 & $\mathrm{dBm}$ \\
\hline$P_{R I S}$ & 10 & $\mathrm{dBm}$ \\
\hline$R_{\min }$ & 0.1 & $\mathrm{bits} / \mathrm{s} / \mathrm{Hz}$ \\
\hline $\min _{U E}$ & 2 & - \\
\hline $\max _{U E}$ & 300 & - \\
\hline $\mathbb{N}$ & 32 & - \\
\hline $\mathbb{K}$ & 16 & - \\
\hline
\end{tabular}

The performance of the proposed algorithm is compared with the plethora of algorithms such as particle swarm optimization (PSO) [29], firefly algorithm (FA) [30], artificial bee colony $(\mathrm{ABC})$ [31], cuckoo search (CS) [32], teaching learning-based optimization (TLBO) [33], basic open-source nonlinear mixed integer programming (BOMIN) [34], social group optimization (SGO) [35], mesh-adaptive-direct-search-algorithm (NOMAD) [36] and genetic algorithm (GA) [37] to compare and validate the results of the proposed algorithm. Figure 5 shows the complete performance analysis of the proposed algorithm. The throughput of the network for all the algorithms is shown in Figure 5a. The highest average $\Omega$ achieved by OAA is $90 \mathrm{Mbps}$. The proposed algorithm starts converging when the number of $U_{E}$ is greater than 180. Figure $5 \mathrm{a}$ also shows that the performance of the OAA is better than other algorithms.

The EE and the latency of the network are also evaluated using several algorithms as shown in Figure $5 b$. Figure $5 b$ shows that the performance of the OAA is better than other algorithms. OAA achieves optimal $\mathbb{E} \mathbb{E}$ of $9 \mathrm{Mb} / \mathrm{J}$ whereas the highest $\mathbb{E} \mathbb{E}$ achieved by the 
other algorithms, particularly NOMAD, is $8.5 \mathrm{Mb} / \mathrm{J}$. OAA shows significant improvement in $\mathbb{E} \mathbb{E}$. The optimal latency achieved by the OAA is $0.5 \mathrm{~ms}$ for $90 U_{E}$, whereas the minimum latency achieved by NOMAD is $1 \mathrm{~ms}$. The performance of NOMAD is better than other algorithms. However, OAA outperforms NOMAD.

We evaluated the channel efficiency $(\mathbb{C E})$ of the RIS channel and NLOS using OAA. Figure $5 c$ shows the $\mathrm{CE}$ in the percentage of OAA with $\Omega$. The $\mathbb{C E}$ of RIS-assisted network is $95 \%$. However, the $\mathbb{C E}$ of the without RIS is $85 \%$ which signifies the importance of RIS surface in the network.

Figure $5 d$, shows the allocation of the RIS to the $U_{E}$. When the number of $U_{E}$ increases, more $U_{E}$ are shifted on RIS to avoid blockage caused by the NLOS. The minimum number of $U_{E}$ that successfully communicates with RIS is 10 . However, when the number of users increases from 10 , the $U_{E}$ is shifted on the RIS channel because the network exceeds the mobile communication network forecast limit. The forecast is predicted using Algorithm 2. The utility improvement makes the network stable and allocates optimal resources to all $U_{E} S$.

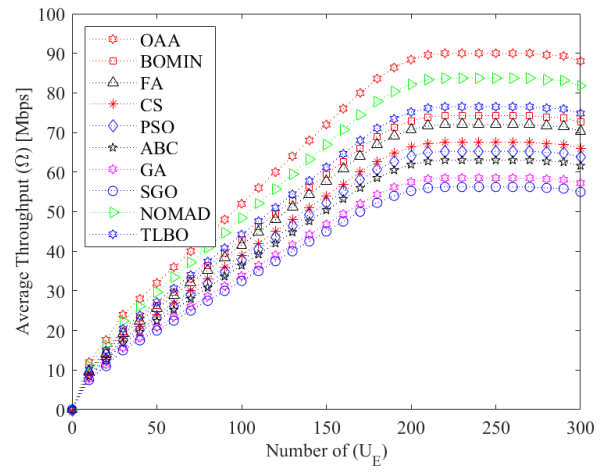

(a)

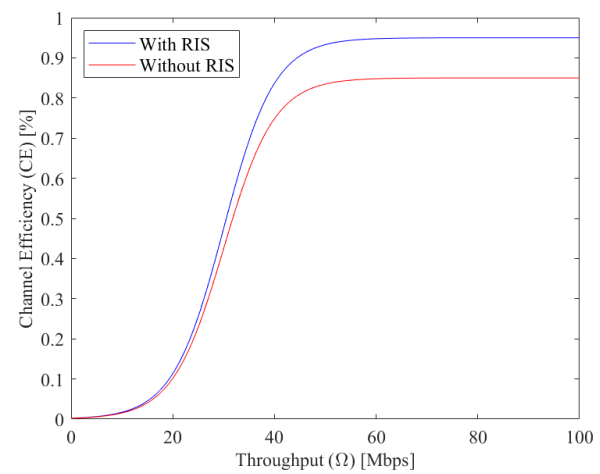

(c)

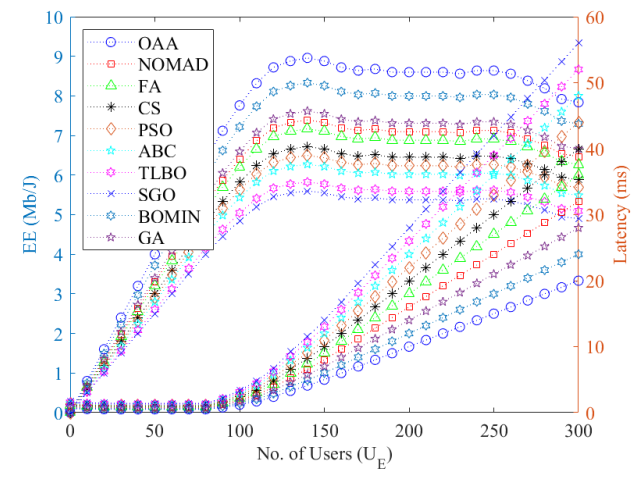

(b)

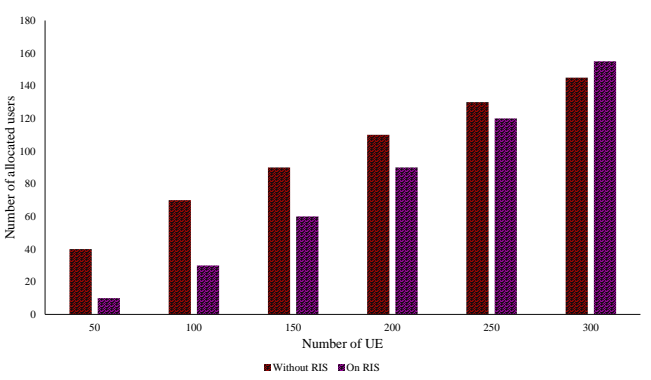

(d)

Figure 5. Performance analysis of the proposed algorithm. (a) Average $\Omega$ (Mbps) vs. No. of $U_{E}$; (b) No. of $U_{E}$ vs. $\mathbb{E} \mathbb{E}$ and Latency (ms); (c) Channel efficiency $(\mathbb{C E})$ vs. $\Omega$; (d) Resource allocation to $U_{E}$.

The upcoming section presents the conclusion.

\section{Conclusions}

Mobile communication is increasing day by day, and the requirement for high $\mathrm{EE}$ and low latency is also growing, particularly in industry 5.0. 6G provides the solution to this problem; however, the deployment of $6 \mathrm{G}$ is challenging. RIS is the primary solution in deploying the 6G, and this article considers an industry scenario and formulates an MINLP optimization problem. We propose an OAA algorithm for the formulated problem. The performance of the proposed algorithm is evaluated in terms of EE, throughput, latency, 
and resource allocation. The optimal EE obtained by OAA is $9 \mathrm{MbJ}^{-1}$, whereas the optimal average $\Omega$ achieved by OAA is $90 \mathrm{Mbps}$. The latency of the network using the OAA algorithm is 0.5 milliseconds. We also compare the performance of the proposed algorithm with several optimization algorithms. The experimental results verify that the proposed OAA outperforms all the other algorithms. In we future, we aim to implement the proposed algorithm in other domains such as in a smart city, heterogeneous networks, human to robots communication.

Author Contributions: Conceptualization, S.J.; Data curation, S.J. and M.S.A.; Formal analysis, S.J. and M.S.A.; Investigation, M.S.A.; Methodology, S.J.; Project administration, M.R.; Supervision, M.R. and F.; Validation, M.R.; Visualization, F.; Writing—original draft, S.J.; Writing—review and editing, M.R. and F. All authors have read and agreed to the published version of the manuscript.

Funding: This research received no external funding.

Institutional Review Board Statement: Not applicable.

Informed Consent Statement: Not applicable.

Data Availability Statement: Not applicable.

Conflicts of Interest: The authors declare no conflict of interest.

\section{References}

1. O'Connell, E.; Moore, D.; Newe, T. Challenges Associated with Implementing 5G in Manufacturing. Telecom 2020, 1, 48-67. [CrossRef]

2. Björnson, E.; Özdogan, Ö.; Larsson, E.G. Intelligent Reflecting Surface Versus Decode-and-Forward: How Large Surfaces are Needed to Beat Relaying? IEEE Wirel. Commun. Lett. 2020, 9, 244-248. [CrossRef]

3. Tekbıyık, K.; Kurt, G.K.; Yanikomeroglu, H. Energy-Efficient RIS-Assisted Satellites for IoT Networks. IEEE Internet Things J. 2021. [CrossRef]

4. $\quad$ Basar, E.; Di Renzo, M.; De Rosny, J.; Debbah, M.; Alouini, M.-S.; Zhang, R. Wireless Communications Through Reconfigurable Intelligent Surfaces. IEEE Access 2019, 7, 116753-116773. [CrossRef]

5. Han, Y.; Tang, W.; Jin, S.; Wen, C.-K.; Ma, X. Large Intelligent Surface-Assisted Wireless Communication Exploiting Statistical CSI. IEEE Trans. Veh. Technol. 2019, 68, 8238-8242. [CrossRef]

6. Huang, C.; Zappone, A.; Alexandropoulos, G.C.; Debbah, M.; Yuen, C. Reconfigurable Intelligent Surfaces for Energy Efficiency in Wireless Communication. IEEE Trans. Wirel. Commun. 2019, 18, 4157-4170. [CrossRef]

7. Di, B.; Zhang, H.; Song, L.; Li, Y.; Han, Z.; Poor, H.V. Hybrid Beamforming for Reconfigurable Intelligent Surface based Multi-User Communications: Achievable Rates with Limited Discrete Phase Shifts. IEEE J. Sel. Areas Commun. 2020, 38, 1809-1822. [CrossRef]

8. Li, L.; Ruan, H.; Liu, C.; Li, Y.; Shuang, Y.; Alù, A.; Qiu, C.-W.; Cui, T.J. Machine-learning reprogrammable metasurface imager. Nat. Commun. 2019, 10, 1082. [CrossRef]

9. Liaskos, C.; Nie, S.; Tsioliaridou, A.; Pitsillides, A.; Ioannidis, S.; Akyildiz, I. A New Wireless Communication Paradigm through Software-Controlled Metasurfaces. IEEE Commun. Mag. 2018, 56, 162-169. [CrossRef]

10. Tan, X.; Sun, Z.; Koutsonikolas, D.; Jornet, J.M. Enabling Indoor Mobile Millimeter-wave Networks Based on Smart Reflect-arrays In Proceedings of the IEEE INFOCOM 2018-IEEE Conference on Computer Communications, Honolulu, HI, USA, 15-19 April 2018; pp. 270-278.

11. Di Renzo, M.; Debbah, M.; Phan-Huy, D.-T.; Zappone, A.; Alouini, M.-S.; Yuen, C.; Sciancalepore, V.; Alexandropoulos, G.C.; Hoydis, J.; Gacanin, H.; et al. Smart radio environments empowered by reconfigurable ai meta-surfaces: An idea whose time has come. EURASIP J. Wireless Commun. Netw. 2019, 2019, 129. [CrossRef]

12. Di Renzo, M.; Zappone, A.; Debbah, M.; Alouini, M.; Yuen, C.; De Rosny, J.; Tretyakov, S. Smart Radio Environments Empowered by Reconfigurable Intelligent Surfaces: How It Works, State of Research, and The Road Ahead. IEEE J. Sel. Areas Commun. 2020, 38, 2450-2525. [CrossRef]

13. Lin, S.; Zheng, B.; Alexandropoulos, G.C.; Wen, M.; Chen, F.; Mumtaz, S. Adaptive Transmission for Reconfigurable Intelligent Surface-Assisted OFDM Wireless Communications. IEEE J. Sel. Areas Commun. 2020, 38, 2653-2665. [CrossRef]

14. Yu, X.; Xu, D.; Schober, R. MISO Wireless Communication Systems via Intelligent Reflecting Surfaces: (Invited Paper). In Proceedings of the 2019 IEEE/CIC International Conference on Communications in China (ICCC), Changchun, China, 11-13 August 2019; pp. 735-740.

15. Basar, E. Transmission Through Large Intelligent Surfaces: A New Frontier in Wireless Communications. In Proceedings of the 2019 European Conference on Networks and Communications (EuCNC), Valencia, Spain, 18-21 June 2019; pp. 112-117.

16. Nadeem, Q.-U.-A.; Kammoun, A.; Chaaban, A.; Debbah, M.; Alouini, M.-S. Asymptotic Max-Min SINR Analysis of Reconfigurable Intelligent Surface Assisted MISO Systems. IEEE Trans. Wirel. Commun. 2020, 19, 7748-7764. [CrossRef] 
17. Buzzi, S.; D'Andrea, C.; Zappone, A.; Fresia, M.; Zhang, Y.-P.; Feng, S. Resource Allocation in Wireless Networks Assisted by Reconfigurable Intelligent Surfaces. In Proceedings of the 2020 IEEE 31st Annual International Symposium on Personal, Indoor and Mobile Radio Communications, London, UK, 31 August-3 September 2020; pp. 1-6.

18. Lee, G.; Jung, M.; Kasgari, A.T.Z.; Saad, W.; Bennis, M. Deep Reinforcement Learning for Energy-Efficient Networking with Reconfigurable Intelligent Surfaces. In Proceedings of the ICC 2020-2020 IEEE International Conference on Communications (ICC), Dublin, Ireland, 7-11 June 2020; pp. 1-6.

19. Yang, Z.; Shi, J.; Li, Z.; Chen, M.; Xu, W.; Shikh-Bahaei, M. Energy Efficient Rate Splitting Multiple Access (RSMA) with Reconfigurable Intelligent Surface. In Proceedings of the 2020 IEEE International Conference on Communications Workshops (ICC Workshops), Dublin, Ireland, 7-11 June 2020; pp. 1-6.

20. Youn, J.; Son, W.; Jung, B.C. Physical-Layer Security Improvement with Reconfigurable Intelligent Surfaces for 6G Wireless Communication Systems. Sensors 2021, 21, 1439. [CrossRef] [PubMed]

21. Odeyemi, K.; Owolawi, P.; Olakanmi, O. Reconfigurable Intelligent Surface in Wireless-Powered Interference-Limited Communication Networks. Symmetry 2021, 13, 960. [CrossRef]

22. Sharma, T.; Chehri, A.; Fortier, P. Reconfigurable Intelligent Surfaces for 5G and beyond Wireless Communications: A Comprehensive Survey. Energies 2021, 14, 8219. [CrossRef]

23. You, L.; Xiong, J.; Ng, D.W.K.; Yuen, C.; Wang, W.; Gao, X. Energy Efficiency and Spectral Efficiency Tradeoff in RIS-Aided Multiuser MIMO Uplink Transmission. IEEE Trans. Signal Process. 2021, 69, 1407-1421. [CrossRef]

24. Ntontin, K.; Di Renzo, M.; Lazarakis, F. On the Rate and Energy Efficiency Comparison of Reconfigurable Intelligent Surfaces with Relays. In Proceedings of the 2020 IEEE 21st International Workshop on Signal Processing Advances in Wireless Communications (SPAWC), Atlanta, GA, USA, 26-29 May 2020; pp. 1-5.

25. Ahsan, M.; Jamil, S.; Ejaz, M.T.; Abbas, M.S. Energy Efficiency Maximization in RIS-assisted Wireless Networks. In Proceedings of the 2021 International Conference on Computing, Electronic and Electrical Engineering (ICE Cube), Quetta, Pakistan, 26-27 October 2021; pp. 1-6.

26. Singh, S.; Kumbhar, A.; Güvenç, İ.; Sichitiu, M.L. Intelligent Interference Management in UAV-Based HetNets. Telecom 2021, 2 , 472-488. [CrossRef]

27. Bennaceur, J.; Ahmadi, H.; Souhi, S. Game-Theoretical Approaches for Service Provisioning in Network Virtualization: Survey, Taxonomies and Open Challenges. Telecom 2021, 2, 232-254. [CrossRef]

28. Le, Q.N.; Nguyen, V.-D.; Dobre, O.A.; Zhao, R. Energy Efficiency Maximization in RIS-Aided Cell-Free Network With Limited Backhaul. IEEE Commun. Lett. 2021, 25, 1974-1978. [CrossRef]

29. Sengupta, S.; Basak, S.; Peters, R.A., II. Particle swarm optimization: A survey of historical and recent developments with hybridization perspectives. Mach. Learn. Knowl. Extr. 2019, 1, 157-191. [CrossRef]

30. Selvakumar, B.; Muneeswaran, K. Firefly Algorithm-Based Feature Selection for Network Intrusion Detection. Comput. Secur. 2019, 81, 148-155.

31. Xu, X.; Hao, J.; Zheng, Y. Multi-Objective Artificial Bee Colony Algorithm for Multi-Stage Resource Leveling Problem in Sharing Logistics Network. Comput. Ind. Eng. 2020, 142, 106338. [CrossRef]

32. Mareli, M.; Twala, B. An Adaptive Cuckoo Search Algorithm for Optimization. Appl. Comput. Inform. 2018, 14, 107-115. [CrossRef]

33. Zou, F.; Chen, D.; Xu, Q. A Survey of Teaching Learning-Based Optimization. Neurocomputing 2019, 335, 366-383. [CrossRef]

34. Cimorelli, L.; Fecarotta, O. Optimal Regulation of Variable Speed Pumps in Sewer Systems. Environ. Sci. Proc. 2020, 2, 2058. [CrossRef]

35. Praveen, S.P.; Rao, K.T.; Janakiramaiah, B. Effective Allocation of Resources and Task Scheduling in Cloud Environment using Social Group Optimization. Arab. J. Sci. Eng. 2018, 43, 4265-4272. [CrossRef]

36. Jamil, S.; Rahman, M.; Tanveer, J.; Haider, A. Energy Efficiency and Throughput Maximization Using Millimeter Waves-Microwaves HetNets. Electronics 2022, 11, 474. [CrossRef]

37. Guerrero, C.; Lera, I.; Juiz, C. Genetic Algorithm for Multi-Objective Optimization of Container Allocation in Cloud Archi-tecture. J. Grid Comput. 2018, 16, 113-135. [CrossRef] 